\title{
ARTIGO ORIGINAL \\ Indicadores Antropométricos de Risco Cardiovascular em Estudantes de Educação Física
}

\author{
Anthropometric Indicators of Cardiovascular Risk in Physical Education Students \\ Evaldo Maciel Pinto ${ }^{1,2}$, Diogo Guimarães ${ }^{3}$, Athos Henrique Rocha Villaça4, Sebastião Lobo da Silva1, Bibiano Madrid5,6. \\ Gilmar Mercês de Jesus, Déborah Santos Conceição \\ Núcleo de Estudos e Pesquisas em Atividade Física e Saúde (NEPAFIS), Universidade Estadual de Feira de Santana, \\ Feira de Santana, Bahia, Brasil
}

\begin{abstract}
Introdução: Entre os fatores de risco para as doenças cardiovasculares (DCV) destaca-se a obesidade, definida como o acúmulo excessivo de gordura corporal, em extensão total ou localizada.

Objetivo: O presente trabalho teve como objetivo descrever os indicadores antropométricos de risco cardiovascular de estudantes de Educação Física da UEFS, conforme características demográficas e comportamentais.

Métodos: Trata-se de um estudo de corte transversal, com uma amostra de 58 estudantes (51,7\% do sexo feminino). Foram descritos o Índice de Massa Corporal (IMC), a Circunferência da Cintura (CC), o Índice de Conicidade (Índice C), e as relações Razão Circunferência Cintura-Quadril (RCCQ) e a Razão Cintura-Estatura (RCEst), conforme sexo, idade, estado civil, ocupação, turno de trabalho, carga horária de trabalho/estágio semanal e estresse percebido. O estudo foi aprovado pelo Comitê de Ética em Pesquisa da UEFS, sob protocolo de número 043/2007.

Resultados: Foi verificado um percentual alto de eutróficos (68,96\%). Apenas a variável sexo apresentou associação significante com o IMC. Os homens apresentaram maior prevalência de sobrepeso e obesidade $(46,4 \%)$ e de Risco Coronariano Elevado segundo o IMC $(64,3 \%)$.

Conclusões: O sexo masculino foi fator de risco para sobrepeso e obesidade. A CC, o Índice C, a RCCQ e a RCEst não apresentaram associação estatisticamente significante com as variáveis estudadas.
\end{abstract}

Palavras-chave: Obesidade. Estudantes. Antropometria.

Introduction: Among the risk factors for CVD is obesity, which can be defined as the excessive accumulation of body fat in total length or located.

Objective: This study aimed to describe the anthropometric indicators of cardiovascular risk students of Physical Education UEFS as demographic and behavioral characteristics.

Methods: The study design was cross-sectional with a sample composed by 58 students $(51.7 \%$ female). This study described the Body Mass Index (BMI), Waist Circumference (WC), the Conicity Index (CI), and relations Waist-to-hip Ratio (WHR) and Waist-to-Height Ratio (WHtR) as sex, age, marital status, occupation, shift work, weekly hours of work and perception of stress. Results: It was found a high percentage of normal, and only the gender variable showed significant association with BMI. Men showed higher prevalence of overweight and obesity (46.4\%) and high coronary risk according to BMI (64.3\%).

Conclusion: The gender male showed like a risk factor for overweight and obesity. The WC, the Cl, and WHR WHtR showed no statistically significant association with variables.

Key-words: Obesity. Students. Anthropometry.

Aceito em: 25/02/2011 - Revista de Educação Física 2012 Ago; 155:19-28. Rio de Janeiro - Brasil

INTRODUÇÃO

As Doenças Crônicas Não-Transmissíveis (DCNT) passaram a liderar as causas de óbitos no Brasil e no mundo, ultrapassando as taxas de mortalidade por Doenças Infecciosas e Parasitárias (DIP). Essas mudanças no perfil de morbidade e mortalidade refletem modificações ocorridas nas condições de habitação e saneamento, alimentação, níveis de ocupação e renda, dinâmica demográfica e estilo de vida da população1.
Estima-se que em torno do ano de 2050 os países emergentes concentrarão a maior população mundial com DCNT e o maior número de óbitos por estas doenças ${ }^{2}$. As DCNT acometem quase $75 \%$ da população brasileira adulta, ocorrendo casos desde a adolescência e são responsáveis por grande parte dos benefícios concedidos pela Previdência Social. Entre as principais responsáveis pela morbidade e mortalidade no grupo das DCNT estão as doenças cardiovasculares (DCV). Elas apresentam uma importante contribuição 
para mortalidade precoce, perda da qualidade de vida e despesas médico-hospitalares ${ }^{2,3}$.

No Brasil, as Doenças do Aparelho Circulatório (DAC) foram responsáveis por $31,46 \%$ dos óbitos por grupo de causa, no ano de 20054. Tem sido reportada a característica perene da alta participação das DAC na mortalidade, sedo que as doenças circulatórias, a isquêmica do coração e a doença cerebrovascular destacam-se com principais causas de morte no Brasil, entre homens e mulheres com idade a partir dos 30 anos ${ }^{5}$.

Entre os fatores de risco não-modificáveis para DCV deve-se considerar a obesidade, definida como o acúmulo excessivo de gordura corporal, em extensão total ou localizada, e relacionada não apenas com a doença cardiovascular, mas ao surgimento de outras doenças como diabetes, hipertensão arterial e certos tipos de câncer6.

A obesidade generalizada relacionada ao acometimento por DCV tem demonstrado uma menor associação ao risco coronariano aumentado do que a obesidade central ou abdominal, avaliada com o auxílio de diversos indicadores e índices antropométricos que, através da avaliação da composição corporal, determinariam essa associação. Entre os mais utilizados podem ser destacados: a Circunferência da Cintura (CC), o Índice de Massa Corporal (IMC), o Índice de Conicidade (IC), e as relações Razão Circunferência Cintura-Quadril (RCCQ) e a Razão Cintura-Estatura (RCEst) ${ }^{7,8}$.

Estudos têm demonstrado uma alta prevalência de fatores de risco cardiovasculares entre populações de adolescentes e adultos jovens, tendo sua agregação um efeito agravante no acometimento por essas doenças. Nestas populações destacam-se entre os fatores de risco o sedentarismo, a obesidade, a hipertensão arterial e as dislipidemias ${ }^{9,12}$.

Nota-se que a identificação do perfil de risco de uma população é um importante instrumento para a elaboração de estratégias para prevenção e controle de doenças cardiovasculares. Para a redução das DCV é essencial a estimativa dos níveis dos fatores de risco convencionais ou estabelecidos para DCV em amostras representativas da população para que sejam identificados os fatores de risco que exigem uma intervenção imediata e avaliação de avaliação dos fatores de risco emergentes, que incluem a obesidade ${ }^{13}$.

Compreendendo a necessidade de ações preventivas na população jovens e sabendo que os indicadores antropométricos são importantes instrumentos para a identificação de risco cardiovascular, esse estudo teve o objetivo de descrever os indicadores antropométricos de risco cardiovascular de estudantes de Educação Física da UEFS, conforme características demográficas e comportamentais.

\section{METODOLOGIA}

Trata-se de um estudo de corte transversal realizado com uma amostra de 58 estudantes (como foi obtida a amostra?), regularmente matriculados entre $\circ 1^{\circ} \mathrm{e} \circ 8^{\circ}$ semestres do curso de Educação Física da Universidade Estadual de Feira de Santana, Bahia.

Nesta pesquisa, as variáveis independentes estudadas foram: o sexo, a idade, estado civil, ocupação, turno de trabalho/estágio, carga horária semanal de trabalho/estágio e percepção de estresse (qual o instrumento utilizado?). As variáveis dependentes estudadas foram os indicadores antropométricos de risco cardiovascular: Índice de Massa Corporal (IMC), CC (Circunferência da Cintura), CA (Circunferência do Abdome), Índice C (Índice de Conicidade), RCQ (Relação Cintura/quadril), e RCE (Relação Cintura/Estatura).

Os dados utilizados nesta pesquisa foram obtidos a partir dos próprios sujeitos (?). Para tanto, foi realizada uma avaliação antropométrica no Laboratório de Atividade Física da Universidade Estadual de Feira de Santana (LAF/UEFS), na qual, foram coletadas informações sobre peso, estatura, circunferência abdominal e de cintura, medidas estas, utilizadas para a construção dos índices antropométricos IMC, CC, RCCQ, RCEst e Índice C.

Foram adotados diferentes pontos de corte para classificar o IMC quanto ao excesso de peso e quanto ao Risco Coronariano Elevado. Indivíduos com IMC $<25 \mathrm{~kg} / \mathrm{m} 2$ foram classificados como com excesso de peso!!!!!!!!!!, segundo classificação sugerida pela Organização Mundial de Saúde. Para o Risco Coronariano Elevado (RCE) foram adotados os pontos de corte sugeridos a partir de um estudo com adultos de Salvador, Bahia ${ }^{14}$, com IMC > $26 \mathrm{~kg} / \mathrm{m} 2$ (mulheres) e com IMC > $24 \mathrm{~kg} / \mathrm{m} 2$ (homens).

Os demais índices antropométricos foram classificados quanto ao Risco Coronariano Elevado, adotando-se, para a RCCQ, os pontos de corte 0,92 para homens e 0,83 para mulheres ${ }^{14}$; para a RCEst os pontos 
de corte 0,52 para homens e 0,53 para mulheres ${ }^{15}$; e, finalmente, para o IC os pontos de corte de 1,25 para homens e 1,18 para mulheres ${ }^{16}$.

Todas as medidas antropométricas foram realizadas em triplicata, estando os sujeitos com roupas adequadas e em posição e condições ideais para as medições. $O$ peso corporal (A massa corporal) foi medido(a) com a utilização de uma balança digital, com precisão de $100 \mathrm{~g}$ e capacidade máxima de $150 \mathrm{Kg}$, estando o estudante em posição ereta, descalço, com os pés unidos, e braços estendidos ao longo do corpo

Para estatura foi utilizado um estadiômetro portátil, desmontável, com plataforma e $216 \mathrm{~cm}$ de altura máxima. Os avaliados estavam com a cabeça erguida, sem adereços na cabeça, descalços, com os pés unidos, braços ao longo do corpo e em apinéia respiratória.

As circunferências da cintura, abdome e quadril foram medidas com auxílio de uma trena antropométrica em acrílico, flexível e não extensível. O estudante permaneceu em posição ereta, com os pés juntos, abdome relaxado, trajando roupas leves e soltas. A circunferência da cintura foi medida na altura média entre o ponto íleo-costal e a ultima costela flutuante, estando a trena em contato direto com a pele. A circunferência do abdome foi medida na altura da cicatriz umbilical, também em contato direto com a pele. A circunferência do quadril foi medida entre o ponto íleo-costal e última costela flutuante, com a trena estendida sem comprimir as partes moles. (citar o protocolo utilizado, exemplo: técnicas descritas por Lohman)

$\mathrm{Na}$ análise dos dados as variáveis foram descritas em termos de freqüência absoluta e relativa, calculandose as razões de prevalência, como medidas de associação e realizando-se o teste do Qui-Quadrado para a significância estatística, com nível de $5 \%$ de significância.

\section{RESULTADOS}

A amostra do estudo foi composta por 58 estudantes $(51,7 \%$ do sexo feminino), entre os quais a maioria possui mais do que 20 anos de idade $(63,8 \%)$, são solteiros $(72,4 \%)$ percepção de estresse de moderado a alto (53,5\%). Neste estudo a renda familiar não foi coletada, mas $63,7 \%$ dos estudantes estagiam ou trabalham/estagiam, cumprindo, em sua maioria, uma carga horária semanal de trabalho/estágio que variou entre $6 \mathrm{e}$
40 horas.

Foi verificado um percentual alto de eutróficos $(68,96 \%)$, segundo a classificação do IMC proposto pela Organização Mundial de Saúde. A maioria das variáveis estudadas não apresentou associação estatisticamente significante com o excesso de peso, apenas a variável sexo (TABELA 1), sendo observada nas mulheres menor prevalência de sobrepeso e obesidade.

No presente estudo, observa-se que o sobrepeso e a obesidade tenderam a ser mais freqüentes entre os estudantes: com mais de 20 anos; casados ou com relacionamento estável; que trabalham/estagiam; com turno misto de trabalho; que referiram percepção de estresse baixo; e que trabalhavam mais de 20 horas semanais (TABELA 1). Contudo, essas associações não alcançaram significância estatística. 
TABELA 1. EXCESSO DE PESO*, CONFORME CARACTERÍSTICAS DEMOGRÁFICAS E COMPORTAMENTAIS.

\begin{tabular}{|c|c|c|c|c|c|c|c|}
\hline \multirow[t]{2}{*}{ Variáveis } & \multicolumn{2}{|c|}{$<25,0 \mathrm{Kg} /$} & \multicolumn{2}{|c|}{$\mathrm{IMC} \geq 25,0 \mathrm{Kg} / \mathrm{m} 2$} & \multirow[b]{2}{*}{$\mathrm{RP}$} & \multirow[b]{2}{*}{ IC } & \multirow[b]{2}{*}{ p-valor } \\
\hline & $\mathrm{n}$ & $\%$ & $\mathrm{n}$ & $\%$ & & & \\
\hline \multicolumn{8}{|l|}{ SEXO $(\mathrm{N}=58)$} \\
\hline Masculino & 15 & 53,6 & 13 & 46,4 & 2,78 & $1,14-6,81$ & 0,0144 \\
\hline Feminino & 25 & 83,3 & 5 & 16,7 & & & \\
\hline \multicolumn{8}{|l|}{ IDADE $(\mathrm{N}=57)$} \\
\hline$\leq 20$ anos & 17 & 81,0 & 4 & 19,0 & & & \\
\hline$>20$ anos & 23 & 63,9 & 13 & 36,1 & 1,90 & $0,71-5,07$ & 0,1744 \\
\hline \multicolumn{8}{|l|}{ ESTADO CIVIL $(n=57)$} \\
\hline Não casado & 30 & 71,4 & 12 & 28,6 & 0,86 & $0,36-2,03$ & 0,7293 \\
\hline Casado/Relacionamento Estável & 10 & 66,7 & 5 & 33,3 & & & \\
\hline \multicolumn{8}{|l|}{ OCUPAÇÃO $(n=57)$} \\
\hline Apenas estuda & 15 & 78,9 & 4 & 21,1 & & & \\
\hline Estágio/Trabalho & 25 & 65,8 & 13 & 34,2 & 1,63 & $0,61-4,31$ & 0,3060 \\
\hline \multicolumn{8}{|l|}{ TURNO DE TRABALHO $(n=35)$} \\
\hline Turno Único & 15 & 78,9 & 4 & 21,1 & & & \\
\hline Turno Misto & 9 & 56,2 & 7 & 43,8 & 2,08 & $0,74-5,84$ & 0,1496 \\
\hline \multicolumn{8}{|l|}{ ESTRESSE PERCEBIDO $(\mathrm{N}=57)$} \\
\hline Baixo & 15 & 57,7 & 11 & 42,3 & 2,19 & $0,94-5,10$ & 0,0592 \\
\hline Moderado/Alto & 25 & 80,6 & 6 & 19,4 & & & \\
\hline \multicolumn{8}{|l|}{$\begin{array}{l}\text { CARGA HORÁRIA DE } \\
\text { TRABALHO SEMANAL }(n=37)\end{array}$} \\
\hline Até $12 \mathrm{~h}$ semanais & 12 & 70,6 & 5 & 29,4 & & & \\
\hline Acima de $12 \mathrm{~h}$ semanais & 12 & 60,0 & 8 & 40,0 & 1,36 & $0,55-3,38$ & 0,5014 \\
\hline
\end{tabular}

${ }^{*}$ Classificação proposta pela Organização Mundial de Saúde (WHO, 2000).

IMC: Índice de Massa Corporal; RP:Razão de Prevalência; IC: Intervalo de Confiança.

Quando o risco coronariano foi descrito segundo IMC (TABELA 2), 62,07\% dos participantes apresentaram baixo risco. Observa-se que a utilização de diferentes pontos de corte não modificou a maior prevalência de risco entre os homens. A maioria dos estudantes do sexo masculino $(64,3 \%)$ foi classificada com risco coronariano elevado (RCE), contra apenas $13,3 \%$ das mulheres. As demais variáveis estudadas não apresentaram associação estatisticamente significante com o RCE segundo IMC.

Os estudantes com estresse baixo apresentaram maior prevalência de risco coronariano elevado, podendo este resultado ser decorrente do fato de o estresse ter sido auto-referido. 
TABELA 2.

RISCO CORONARIANO ELEVADO (RCE) AVALIADO PELO IMC*, SEGUNDO CARACTERÍSTICAS DEMOGRÁFICAS E COMPORTAMENTAIS.

\begin{tabular}{|c|c|c|c|c|c|c|c|}
\hline \multirow[t]{2}{*}{ Variáveis } & \multicolumn{2}{|c|}{ Baixo Risco } & \multicolumn{2}{|c|}{ RCE } & \multirow[b]{2}{*}{$\mathrm{RP}$} & \multirow[b]{2}{*}{ IC } & \multirow[b]{2}{*}{ p-valor } \\
\hline & $\mathrm{N}$ & $\%$ & $\mathrm{n}$ & $\%$ & & & \\
\hline \multicolumn{8}{|l|}{ SEXO $(N=58)$} \\
\hline Masculino & 10 & 35,7 & 18 & 64,3 & 4,82 & $1,86-12,51$ & 0,0001 \\
\hline Feminino & 26 & 86,7 & 4 & 13,3 & & & \\
\hline \multicolumn{8}{|l|}{ IDADE $(\mathrm{N}=57)$} \\
\hline$\leq 20$ anos & 15 & 71,4 & 6 & 28,6 & & & \\
\hline$>20$ anos & 21 & 56,8 & 16 & 43,2 & 1,51 & $0,70-3,27$ & 0,2684 \\
\hline \multicolumn{8}{|l|}{ ESTADO CIVIL ( $\mathrm{N}=57)$} \\
\hline Não casado & 28 & 66,7 & 14 & 33,3 & 1,40 & $0,70-2,79$ & 0,3581 \\
\hline Casado/Relacionamento Estável & 8 & 53,3 & 7 & 46,7 & & & \\
\hline \multicolumn{8}{|l|}{ OCUPAÇÃO (N=57) } \\
\hline Apenas estuda & 13 & 68,4 & 6 & 31,6 & & & \\
\hline Estágio/Trabalho & 23 & 60,5 & 15 & 39,5 & 1,25 & $0,58-2,70$ & 0,5602 \\
\hline \multicolumn{8}{|l|}{ TURNO DE TRABALHO (N=35) } \\
\hline Turno Único & 14 & 73,7 & 5 & 26,3 & & & \\
\hline Turno Misto & 7 & 43,8 & 9 & 56,2 & 2,14 & $0,90-5,09$ & 0,0717 \\
\hline \multicolumn{8}{|l|}{ ESTRESSE PERCEBIDO $(\mathrm{N}=57)$} \\
\hline Baixo & 12 & 46,2 & 14 & 53,8 & 2,38 & $1,13-5,01$ & 0,0148 \\
\hline Moderado/Alto & 24 & 77,4 & 7 & 22,6 & & & \\
\hline \multicolumn{8}{|l|}{$\begin{array}{l}\text { CARGA HORÁRIA DE } \\
\text { TRABALHO SEMANAL (N=37) }\end{array}$} \\
\hline Até $12 \mathrm{~h}$ semanais & 11 & 64,7 & 6 & 35,3 & & & \\
\hline Acima de $12 \mathrm{~h}$ semanais & 11 & 55,0 & 9 & 45,0 & 1,27 & $0,57-2,85$ & 0,5490 \\
\hline
\end{tabular}

*Os pontos de corte adotados para IMC foram $24,0 \mathrm{Kg} / \mathrm{m} 2$ para homens e $26,0 \mathrm{Kg} / \mathrm{m} 2$ para mulheres (PITANGA; LESSA, 2005). RCE: Risco coronariano Elevado; RP: Razão de Prevalência; IC: Intervalo de Confiança 
TABELA 3.

RISCO CORONARIANO AUMENTADO AVALIADO PELA CIRCUNFERÊNCIA DA CINTURA (CC $)^{*}$, SEGUNDO CARACTERÍSTICAS DEMOGRÁFICAS E COMPORTAMENTAIS.

\begin{tabular}{|c|c|c|c|c|c|c|c|}
\hline \multirow[t]{2}{*}{ Variáveis } & \multicolumn{2}{|c|}{ Baixo Risco } & \multicolumn{2}{|c|}{ Risco Aumentado } & \multirow[b]{2}{*}{ RP } & \multirow[b]{2}{*}{ IC } & \multirow[b]{2}{*}{ p-valor } \\
\hline & $\mathrm{n}$ & $\%$ & $\mathrm{n}$ & $\%$ & & & \\
\hline \multicolumn{8}{|l|}{$\operatorname{SEXO}(\mathrm{N}=58)$} \\
\hline Masculino & 21 & 75,0 & 7 & 25,0 & 3,75 & & \\
\hline Feminino & 28 & 93,3 & 2 & 6,7 & & $0,85-16,55$ & 0,05398 \\
\hline \multicolumn{8}{|l|}{ IDADE $(\mathrm{N}=57)$} \\
\hline$\leq 20$ anos & 18 & 85,7 & 3 & 14,3 & 1,05 & & \\
\hline$>20$ anos & 31 & 83,8 & 6 & 16,2 & & $0,63-1,75$ & 0,8452 \\
\hline \multicolumn{8}{|l|}{ ESTADO CIVIL (N=57) } \\
\hline Não casado & 35 & 83,3 & 7 & 16,7 & 1,07 & $0,72-1,57$ & 0,7612 \\
\hline Casado/Relacionamento Estável & 13 & 86,7 & 2 & 13,3 & & & \\
\hline \multicolumn{8}{|l|}{ OCUPAÇÃO (N=57) } \\
\hline Apenas estuda & 17 & 89,5 & 2 & 10,5 & & & \\
\hline Estágio/Trabalho & 31 & 81,6 & 7 & 18,4 & 1,75 & $0,40-7,63$ & 0,4409 \\
\hline \multicolumn{8}{|l|}{ TURNO DE TRABALHO $(\mathrm{N}=35)$} \\
\hline Turno Único & 17 & 89,5 & 2 & 10,5 & & & \\
\hline Turno Misto & 11 & 68,8 & 5 & 31,3 & 2,97 & $0,66-13,29$ & 0,1267 \\
\hline \multicolumn{8}{|l|}{ ESTRESSE $(\mathrm{N}=57)$} \\
\hline Baixo & 21 & 80,8 & 5 & 19,2 & 1,49 & $0,45-4,98$ & 0,5141 \\
\hline Moderado/Alto & 27 & 87,1 & 4 & 12,9 & & & \\
\hline \multicolumn{8}{|l|}{$\begin{array}{l}\text { CARGA HORÁRIA DE } \\
\text { TRABALHO SEMANAL (N=37) }\end{array}$} \\
\hline Até $12 \mathrm{~h}$ semanais & 14 & 82,4 & 3 & 17,6 & & & \\
\hline Acima de $12 \mathrm{~h}$ semanais & 16 & 80,0 & 4 & 20,0 & 1,13 & $0,29-4,37$ & 0,8555 \\
\hline
\end{tabular}

* Os pontos de corte adotados para Circunferência da Cintura foram CC $>83 \mathrm{~cm}$ para mulheres e $\mathrm{CC}>88 \mathrm{~cm}$ para homens (PITANGA; LESSA, 2005). Estresse: Percepção de Estresse, RP: Razão de Prevalência; IC: Intervalo de Confiança.

A Razão Cintura-Estatura não apresentou associação estatisticamente significante com as variáveis estudadas, o que indica não haver casos de obesidade abdominal significativa entre os estudantes.

As baixas prevalências de risco aumentado segundo a RCEst reflete os valores absolutos baixos de CC entre os estudantes já que esta medida apresenta relação diretamente proporcional aos valores deste indicadores de obesidade abdominal. 
TABELA 4.

RISCO CORONARIANO AUMENTADO AVALIADO PELA RAZÃO CINTURAESTATURA (RCEST)*, SEGUNDO CARACTERÍSTICAS DEMOGRÁFICAS E COMPORTAMENTAIS.

\begin{tabular}{|l|l|l|l|l|l|l|l|}
\hline & \multicolumn{2}{l|}{ Vaixo Risco } & \multicolumn{2}{l|}{ Risco Aumentado } & & & \\
\hline & $\mathrm{n}$ & $\%$ & $\mathrm{n}$ & $\%$ & $\mathrm{RP}$ & $\mathrm{IC}$ & $\mathrm{p}$-valor \\
\hline SEXO (N=58) & & & & & & & \\
\hline Masculino & 25 & 89,3 & 3 & 10,7 & 1,61 & $0,29-8,92$ & 0,5831 \\
\hline Feminino & 28 & 93,3 & 2 & 6,7 & & & \\
\hline IDADE (N=57) & & & & & & & \\
\hline$\leq 20$ anos & 19 & 90,5 & 2 & 9,5 & & & \\
\hline$>20$ anos & 33 & 91,7 & 3 & 8,3 & 0,88 & $0,16-4,82$ & 0,8782 \\
\hline ESTADO CIVIL (N=57) & & & & & & & \\
\hline Não casado & 38 & 90,5 & 4 & 9,5 & 1,43 & $0,17-11,79$ & 0,7370 \\
\hline Casado/Relacionamento Estável & 14 & 93,3 & 1 & 6,7 & & & \\
\hline OCUPAÇÃO (N=57) & & & & & & & \\
\hline Apenas estuda & 18 & 94,7 & 1 & 5,3 & & & \\
\hline Estágio/Trabalho & 34 & 89,5 & 4 & 10,5 & 2,00 & $0,24-16,68$ & 0,5079 \\
\hline TURNO DE TRABALHO (N=35) & & & & & & & \\
\hline Turno Único & 18 & 94,7 & 1 & 5,3 & & & \\
\hline Turno Misto & 13 & 81,3 & 3 & 18,7 & 3,56 & $0,41-30,99$ & 0,2115 \\
\hline ESTRESSE (N=57) & & & & & & & \\
\hline Baixo & 23 & 88,5 & 3 & 11,5 & 1,79 & $0,32-9,90$ & 0,4989 \\
\hline Moderado/Alto & 29 & 93,5 & 2 & 6,5 & & & \\
\hline $\begin{array}{l}\text { CARGA HORÁRIA DE } \\
\text { TRABALHO SEMANAL (N=37) }\end{array}$ & & & & & & & \\
\hline Até 12h semanais & 16 & 94,1 & 1 & 5,9 & & & \\
\hline Acima de 12h semanais & 17 & 85,0 & 3 & 15,0 & 2,55 & $0,29-22,31$ & 0,3734 \\
\hline
\end{tabular}

${ }^{*}$ Os pontos de corte para RCEst adotados foram 0,52 para homens e 0,53 para mulheres (PITANGA; LESSA, 2006). RP: Razão de Prevalência; IC: Intervalo de Confiança. 


\section{DISCUSSÃo}

O alto percentual de estudantes eutróficos encontrado foi semelhante a resultados ao de outros estudos em estudantes universitários pelo Brasil ${ }^{17-20}$.

Note-se que tem sido comentado o desempenho similar para indicação do estado nutricional e obesidade abdominal ao se analisar a concordância e eficiência de diferentes valores de ponto de corte para IMC em adolescentes ${ }^{21}$. Comentar os pontos de corte e comparar com os utilizados no estudo.

A análise dos resultados revelou que a maioria das variáveis estudadas não apresentou associação estatisticamente significante com o excesso de peso, apenas a variável sexo, sendo observada nas mulheres menor prevalência de sobrepeso e obesidade.

Isto foi semelhante a reportado em um estudo que descreveu a prevalência de fatores de risco para doenças cardiovasculares em estudantes da área de saúde da Universidade Federal de Pernambuco, no qual foi encontrada uma prevalência de excesso de peso de $35,5 \%$ no sexo masculino e 5,3\% no feminino ${ }^{22}$. Entretanto chamou-se a atenção para a possibilidade de os resultados mascararem o verdadeiro estado nutricional dos estudantes pernambucanos, devido a distúrbios do comportamento alimentar relacionados ao receio de engordar, freqüentes em mulheres jovens e, por outro lado, à busca por um corpo musculoso, também, comum entre homens jovens. (não foi feita uma investigação que pudesse fundamentar tal afirmação!!! A prevalência da bulemia nervosa é de, aproximadamente, $1 \%$ da população de mulheres jovens; as síndromes de transtornos alimentares parciais ocorrem em $2 \%$ a $5 \%$ das mulheres jovens. A anorexia nervosa provavelmente ocorre em menos de $0,5 \%$ dessa população. Portanto, o baixo percentual identificado no sexo feminino não está relacionado com os transtornos alimentares.)

De fato, por se tratar de uma população semelhante, a associação do sexo com o excesso de peso entre os estudantes de Educação Física da UEFS, pode ter sofrido o mesmo efeito. (reconsiderar a afirmação pelos motivos acima descritos)

Os estudantes com estresse baixo apresentaram maior prevalência de risco coronariano elevado, podendo este resultado ser decorrente do fato de o estresse ter sido auto-referido. Esse resultado contradiz dados da literatura, que apresentam o estresse como um fator de risco psicológico para o desenvolvimento de doença cardiovascular, e afirma que uma alta exposição ao estresse causaria o aumento de outros fatores de risco, incluindo a obesidade ${ }^{23}$.

Quanto aos resultados encontrados para Circunferência da Cintura, nota-se que os participantes, majoritariamente, apresentaram baixo risco coronariano $(84,48 \%)$. Os homens apresentaram maior prevalência de risco aumentado, apesar de nenhuma variável apresentar associação estatisticamente significante com o risco coronariano elevado.

Tem sido observado, entre os homens, aumento da prevalência de hipertensão arterial com o aumento da CC. Em mulheres, a prevalência da hipertensão aumenta tanto para maiores valores de IMC como de CC. Esses resultados levaram à conclusão de que a obesidade abdominal tem maior impacto sobre a hipertensão entre homens, enquanto que nas mulheres tanto a obesidade central como a generalizada relacionam-se ao aumento da hipertensão24.

Além disso, reportam-se maiores prevalências de obesidade abdominal em mulheres adultas, conforme a medida da circunferência da cintura ${ }^{25}$, resultado oposto ao encontrado no presente estudo.

Nesse sentido, com a utilização do valor de $86 \mathrm{~cm}$ como ponto de corte da CC para estimar a prevalência de obesidade abdominal em funcionárias de uma instituição de ensino superior baiana, foram observados $37,3 \%$ de prevalência de obesidade abdominal ${ }^{26}$.

As baixas prevalências de risco aumentado segundo a RCEst reflete os valores absolutos baixos de CC entre os estudantes já que esta medida apresenta relação diretamente proporcional aos valores deste indicadores de obesidade abdominal.

Ademais, deve ser considerado o fato de este resultado ser devido a uma população adulta jovem, pois é encontrada na literatura referência a associação positiva da obesidade abdominal com a idade, com o seu aumento influenciado pelo aumento da idade ${ }^{27,28}$.

\section{CONCLUSÃO}

A prevalência de sobrepeso e obesidade foi baixa entre os estudantes, podendo ser reflexo do grande de número de participantes jovens.

Os casos de obesidade encontrados neste estudo foram mais freqüente entre os 
indivíduos do sexo masculino, resultado oposto ao encontrado para a população brasileira. A obesidade abdominal foi pouco freqüente e os homens também apresentaram maior freqüência de risco aumentado quando descritos os indicadores de obesidade abdominal, porém essa relação não apresentou significância estatística.

A Razão Cintura-Quadril, o Índice de Conicidade e a Razão Cintura-Estatura não apresentaram associação estatisticamente significante com as variáveis estudadas, refletindo os baixos valores absolutos da Circunferência da Cintura (CC) e a baixa ocorrência encontrada para risco aumentado segundo a CC.

No presente estudo a maioria dos indicadores antropométricos de risco cardiovascular descritos não demonstrou associação estatisticamente significante com as variáveis preditoras.

Os achados desta pesquisa apontam para a necessidade da realização de novos estudos uma amostramaissignificativadapopulaçãouniversitária, e seja feita a utilização de outras variáveis e de outros fatores relacionados às doenças carviovasculares.

\section{REFERÊNCIAS BIBLIOGRÁFICAS}

1. Batista Filho M, Rissin A. A transição nutricional no Brasil: tendências regionais e temporais. Cad Saúde Pública. 2003; 19(1): 181-191.

2. Lessa I. Doenças crônicas não-transmissíveis no Brasil: um desafio para a complexa tarefa da vigilância. Ciênc Saúde Coletiva. 2004; 9(4):931-943.

3. Moura AAG, Carvalho EF, Silva NJC. Repercussão das doenças crônicas não-transmissíveis na concessão de benefícios pela previdência social. Ciênc Saúde Coletiva. 2007; 12(6):1661-1672.

4. Brasil. Datasus. 2009. Disponível em:<http:// tabnet.datasus.gov.br/cgi/tabcgi.exe?idb2007/c04. def>[2008 jul 15].

5. Mansur AP, Favarato D, Souza MFM, Avakian SD, Aldrighi JM, César LAM, Ramires JAF. Tendência do Risco de Morte por Doenças Circulatórias no Brasil de 1979 a 1996. Arq Bras Cardiol. 2001; 76(6):497-503.

6. Pinheiro ARO, Freitas SFT, Corso ACT. Uma abordagem epidemiológica da obesidade. Rev Nutr. 2004; 17(4):523-533.
7. Pitanga FJG, Lessa I. Associação entre indicadores antropométricos de obesidade e risco coronariano em adultos na cidade de Salvador, Bahia, Brasil. Rev Bras Epidemiol. 2007; 10(2): 239-248.

8. Acuña K, Cruz T. Avaliação do estado nutricional de adultos e idosos e situação nutricional da população brasileira. Arq Bras Endocrinol Metab. 2004; 48 ${ }^{(3)}$ : 345361.

9. Nobre MRC, Domingues RZL, Silva AR, Colugnati FAB, Taddei JAAC. Prevalências de sobrepeso, obesidade e hábitos de vida associados ao risco cardiovascular em alunos do ensino fundamental. Rev Assoc Med Bras. 2006; 52(2):118-124.

10. Romaldini CC, Issler $\mathrm{H}$, Cardoso AL, Diament $\mathrm{J}$, Forti N. Fatores de risco para aterosclerose em crianças e adolescentes com história familiar de doença arterial coronariana prematura. J Pediatr. 2004; 80(2): 135-140.

11. Coelho VG, Caetano LF, Liberatore Júnior RDR, Cordeiro JÁ, Souza DRS. Perfil lipídico e fatores de risco para doenças cardiovasculares em estudantes de medicina. Arq Bras Cardiol. 2005; 85(1): 57-62.

12. Brandão AA, Magalhães MEC, Freitas EV, Pozzan R, Brandão AP. Prevenção da doença cardiovascular: a aterosclerose se inicia na infância? Rev SOCERJ. 2004; 17(1): 37-44.

13. Avezum A, Rossi Neto JM, Piegas L. Why do we need randomized and epidemiological studies on cardiovascular disease? evidence-based cardiology VII. Arq Bras Cardiol. 1999; 72(3): 289-295.

14. Pitanga FJG, Lessa I. Indicadores antropométricos de obesidade como instrumento de triagem para risco coronariano elevado em adultos na cidade de Salvador - Bahia. Arq Bras Cardiol. 2005; 85(1): 26-31.

15. Pitanga FJG, Lessa I. Razão cintura-estatura como discriminador do risco coronariano de adultos. Rev Assoc Med Bras. 2006; 52(3): 157-161.

16. Pitanga FJG, Lessa I. Sensibilidade e especificidade do índice de conicidade como discriminador do risco coronariano de adultos em Salvador, Brasil. Rev Bras Epidemiol. 2004; 7(3): 259269.

17. Arechabaleta G, Castillo H. Herrera H, Pacheco M. Composición corporal en una población de estudiantes universitarios. RFM. 2002; 25(2): 209216. 
18. Vieira VCR, Priore SE, Ribeiro SMR, Franceschini SCC, Almeida LP. Perfil socioeconômico, nutricional e de saúde de adolescentes recém-ingressos em uma universidade pública brasileira. Rev Nutr. 2002; 15(3): 273-282.

19. Bion FM, Chagas MHC, Muniz GS, Sousa GLO. Estado nutricional, medidas antropométricas, nivel socioeconómico y actividad física em universitarios brasileños. Nutr Hosp. 2008; 23(3): 234-241.

20. Vargas-Zárate $M$, Becerra-Bulla $F$, PrietoSuárez E. Evaluación antropométrica de estudiantes universitarios en Bogotá, Colombia. Rev de Salud Pública.2008; 10(3): 433-442.

21. Fernandes RA, Rosa CSC, Silva CB, Bueno DR, Oliveira AR, Freitas Júnior IFF. Desempenho de diferentes valores críticos de Índice de Massa Corporal na identificação de excesso de gordura corporal e obesidade abdominal em adolescentes. Rev Assoc Med Bras. 2007; 53(6): 515-519.

22. Petribú MMV. Consumo alimentar e risco de doença cardiovascular em universitários. [Dissertação de Mestrado - Programa de Pós-Graduação em Nutrição] Recife (PE): Universidade Federal de Pernambuco; 2008.

23. Cantos GA, Duarte MFS, Dutra RL, Silva CSM, Waltrick CDA, Balen, MG et al. Prevalência de fatores de risco de doença arterial coronária em funcionários de hospital universitário e sua correlação com estresse psicológico. J Bras Patol Med Lab. 2004; 40(4): 240-247.

24. Peixoto MRG, Benício MHD, Latorre MRDO, Jardim PCBV. Circunferência da cintura e índice de massa corporal como preditores da hipertensão arterial. Arq Bras Cardiol. 2006; 87: 462-470.

25. Olinto MTA, Nácul LC, Dias-Da-Costa JS, Gigante DP, Menezes AMB, Macedo S. Níveis de intervenção para obesidade abdominal: prevalência e fatores associados. Cad Saúde Pública. 2007; 22(6): 1207-1215.

26. Almeida RT, Almeida MMG, Araújo TM. Obesidade abdominal e risco cardiovascular: desempenho de indicadores antropométricos em mulheres. Arq Bras Cardiol. 2009; 92(5): 375-380.

27. Machado PAN, Sichieri R. Relação cinturaquadril e fatores de dieta em adultos. Rev Saúde Pública. 2002; 36(1): 108-204.
28. Gigante DP, Barros FC, Post CLA, Olinto MTA. Prevalência de obesidade em adultos e seus fatores de risco. Rev Saúde Pública. 1997; 31(3): 236-246

Endereço para corespondência:

GILMAR MERCÊS DE JESUS, Rua Juiz de Fora, 581,

Caseb, Feira de Santana, Bahia, CEP: 44052-072, tel.: 75 3225-3477, e-mail: gilmj@yahoo.com.br.

Telefone para contato: (61) 9151-6081 - José Fernando Vila Nova de Moraes 